\title{
Hierarchical Vortex Regions in Swirling Flow
}

\author{
C. Petz, J. Kasten, S. Prohaska and H.-C. Hege \\ Zuse Institute Berlin (ZIB), Germany
}

\begin{abstract}
We propose a new criterion to characterize hierarchical two-dimensional vortex regions induced by swirling motion. Central to the definition are closed loops that intersect the flow field at a constant angle. The union of loops belonging to the same area of swirling motion defines a vortex region. These regions are disjunct but may be nested, thus introducing a spatial hierarchy of vortex regions. We present a parameter free algorithm for the identification of these regions. Since they are not restricted to star- or convex-shaped geometries, we are able to identify also intricate regions, e.g., of elongated vortices. Computing an integrated value for each loop and mapping these values to a vortex region, introduces new ways for visualizing or filtering the vortex regions. Exemplary, an application based on the Rankine vortex model is presented. We apply our method to several CFD datasets and compare our results to existing approaches.
\end{abstract}

Categories and Subject Descriptors (according to ACM CCS): Computer Graphics [I.3.8]: Applications—Physical Sciences and Engineering [J.2]: Physics-

\section{Introduction}

Understanding the structure of vortex systems is an important task in flow analysis. Flow analysis with CFD or experimental methods has become routine in many technical engineering fields. Vortices belong to the most important structures in flow fields. They are often directly connected to the objective of optimization in engineering problems. For instance, vortical structures directly impact the lift of airfoils and they are tightly connected to cavitation effects on marine propulsors. Besides the pure existence and location of vortices, also their areas of influence are of interest for a detailed comprehension of the flow structure. Vortices interact with each other and evolve over time. Formation and collapsing of vortices, growing and shrinking, as well as merging and pairing are important events that characterize the flow. In summary, the whole system consisting of vortices and their interconnections, as well as vortex strengths, sizes, and extents are important for understanding flow situations.

Despite the importance of vortices, a commonly accepted definition of a vortex including its spatial extent and a unique characterization of vortex hierarchies does not exist. Various criteria are used in practice to find and visualize systems of vortices. Among them, vortex definitions based on swirling motion of the velocity field of a flow solution are widely

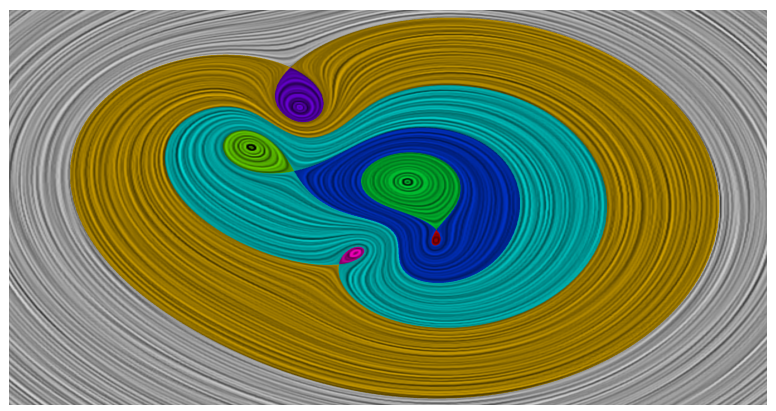

Figure 1: Hierarchical vortex regions resulting from a superposition of Oseen vortices. Distinct vortex regions are colored differently.

used. Although the velocity field is not Galilean invariant and thus dependent on the frame of reference, swirling motion is illustrative and complies well with an intuitive notion of vortices. The shape of a vortex region depends on the chosen vortex criterion. For the analysis of swirling motion vortices, a criterion that is able to identify complex regions of weak and elongated vortices reliably is desirable. Vortex merging and splitting can create complex systems of vortices 
that are best described hierarchically. An automatic extraction of vortex hierarchies therefore can give valuable insight to vortex evolution.

In this paper we present a novel definition of $2 \mathrm{D}$ vortex regions of swirling motion type, based on the angle between the local flow direction and the region's boundary. We further describe an algorithm to identify such regions and to extract them in a hierarchical fashion. The extracted regions comply well with an intuitive understanding of swirling motion. The key advantages of this method are:

- the extraction algorithm is parameter-free,

- arbitrarily shaped, smooth vortex regions are found (not just star-shaped regions),

- hierarchical vortex structures are detected,

- vector fields with and without divergence are handled alike, and

- the method is grid-independent: only streamline tracing and critical point detection are needed.

\section{Related Work}

Vortex visualization and extraction has been broadly discussed; a good overview has been presented by Post et al. [PVH $\left.{ }^{*} 03\right]$. Three main approaches for the extraction of vortex geometries exist: methods that are based purely on properties of a physical indicator function, methods that require also a geometrical construction, and purely geometrical methods.

Indicator based extraction methods first compute a scalar valued indicator function, whose magnitude relates to the strength of vortex activity. Commonly used indicator quantities are vorticity magnitude, pressure, helicity, normalized helicity, $\lambda_{2}$ by Jeong et al. [JH95] or the quantity Q by Hunt [Hun87]. In order to obtain geometrical representations of vortex regions, iso-surfaces of these indicator functions are computed. Methods using this approach often do not identify individual vortices and are usually not able to separate adjacent vortices. Furthermore, the extracted regions depend on a parameter that is often chosen arbitrarily, the threshold value of the iso-surfaces. Schneider et al. [SWC*08] extracted vortex regions based on $\lambda_{2}$; they used a contour tree for the selection of the geometry.

Another approach for determining the shape of a vortex is to explicitly extract the vortex hull geometry for each given or previously extracted vortex coreline by sending out a fan of rays. All these methods, made for star-shaped regions, have been defined on 2D slices of a given vector field. Banks and Singer [BS95] introduced this method to construct a vortex hull as a series of connected contour lines. A fan of rays emanating from the core is sent out, until a threshold of a vortex region criterion is exceeded. The contour line that connects the endpoints of the rays is the outer boundary of the vortex in that plane. As vortex criterion they used a pressure threshold. Bauer et al. [BPSS02] used the same method and a threshold of the absolute value of the imaginary part of the complex eigenvalues of the velocity gradient tensor as vortex region criterion. Stegmaier et al. [SRE05] used a $\lambda_{2}$ threshold for the termination of the sampling rays. Sharing the same basic idea, Garth et al. [GTS*04] extended that approach. Motivated by the Rankine vortex model, they searched for local maxima of the tangential velocity component. Jankun-Kelly et al. [JKJTM06] used the same method for the vortex hull extraction and Tricoche et al. [TGK $\left.{ }^{*} 04\right]$ for analyzing complex datasets.

A purely geometric approach was chosen by Sadarjoen and Post [SP99]. In 2D steady flow fields a search for streamlines with a winding angle of $2 \pi$ was performed with the additional requirement that the distance between start and endpoint of a streamline is relatively close. Streamlines that belong to the same vortex were clustered and an elliptic representation was computed from all sample points of the streamlines. This approach was extended by Reinders et al. [RSVP02] for 3D vortices with known vortex core axis direction.

Bauer and Peikert [BP02] presented a method for vortex coreline extraction and tracking in scale-space. Feature lines break at smaller scales and thus introduce a hierarchy of vortices.

Wischgoll et al. [WS01] first introduced an extraction algorithm for closed streamlines, further improvements were made for example by Theisel et al. [TWHS04] by introducing a grid-independent algorithm. Vector field topology, introduced by Helman and Hesselink [HH89, HH91] and Asimov [Asi93] is the basis of most work for structural analysis of vector fields. We generalize the concept of closed streamline loops and present an algorithm for vortex region extraction that makes use of vector field topology.

\section{Vortex Regions}

We propose a definition for the shape of a vortex region that is motivated by closed streamlines of divergence-free vector fields. In the intuitive understanding of a vortex of a divergence-free steady flow field, particles move around a center, e.g. a vortex consists of the union of all closed orbits winding around a common center.

In vector fields with divergence, streamlines are not closed. As a generalization of the streamline criterion, we introduce lines whose tangents have a constant incident angle to the vector field. Such lines are illustrated in Fig. 2. From top left to bottom right the four images show linear vector fields with increasing divergence and decreasing curl.

This angle criterion removes the divergence in a vortex region by decomposing the vector field in components tangential and perpendicular to a line of constant incident angle. The former is divergence-free, the latter is curl-free. This is similar to a Helmholtz-Hodge decomposition [Hel58] of the 

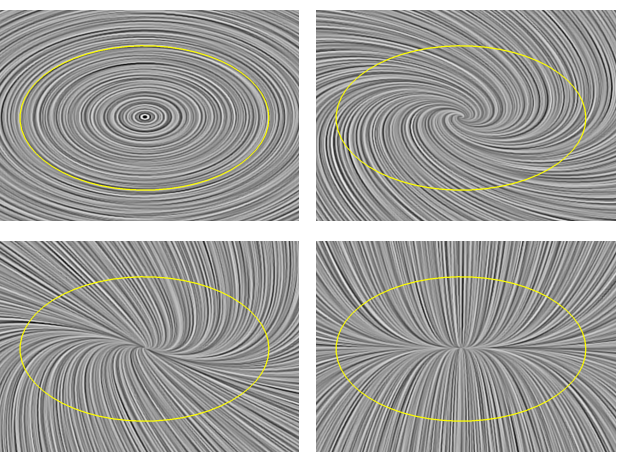

Figure 2: Linear vector fields from divergence-free (topleft) to curl-free (bottom-right), created by rotating vectors of a divergence-free field up to $\pi / 2$. The yellow ellipses intersect the vector fields in a constant angle.

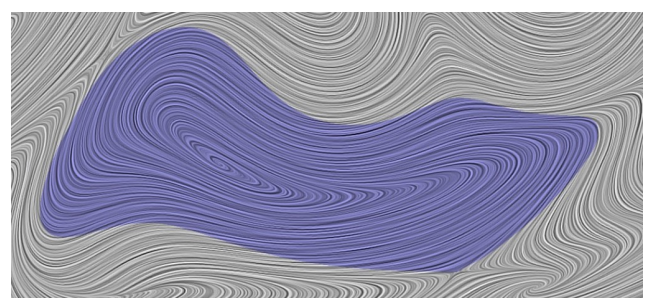

(a) Vortex Region

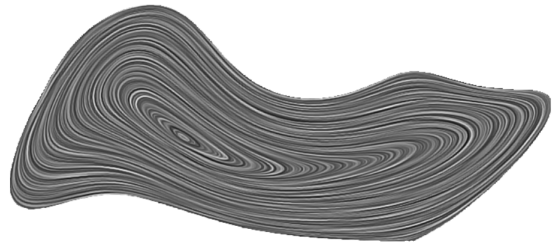

(b) Divergence-free

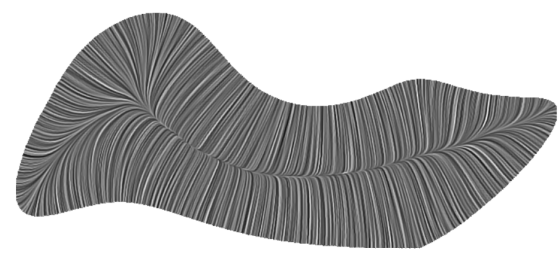

(c) Curl-free

Figure 3: Complex-shaped vortex region with divergence (a), streamlines converge towards the critical point. Vector field decomposition induced by the lines of constant incident angle in a divergence-free (b) and curl-free part (c).

vector field, but for each vortex region separately, instead of the entire field. Such a decomposition is depicted in Fig. 3 for a non-trivial vortex. The lines with constant incident angle are streamlines in the divergence-free vector field.

\subsection{Definition}

We now formalize our motivation. We start from a given continuous $2 \mathrm{D}$ vector field

$$
V: \mathbb{R}^{2} \rightarrow \mathbb{R}^{2},
$$

and apply the rotation operator $R_{\phi}$, which rotates the field by an angle of $\phi$ in counter-clockwise direction, i.e.

$$
R_{\phi}=\left(\begin{array}{cc}
\cos \phi & -\sin \phi \\
\sin \phi & \cos \phi
\end{array}\right) .
$$

Vector fields rotated by $\phi$ are denoted as

$$
V_{\phi}=R_{\phi} V .
$$

A positively oriented closed streamline in the vector field $V_{\phi}$ is called loop $\gamma_{\phi}$ of $V$. We explicitly exclude (limiting) loops passing through critical points. Such a loop $\gamma_{\phi}$ intersects the original vector field at a constant angle, i.e.

$$
\Varangle\left(V\left(\gamma_{\phi}(t)\right), \dot{\gamma}_{\phi}(t)\right)=\phi,
$$

since rotating the vectors around that angle yields a vector field tangential to the loop. For a vector field $V$, the set $M(V)$ of all loops is defined as

$$
M(V)=\left\{\gamma_{\phi} \mid \phi \in[0,2 \pi], \gamma_{\phi} \text { loop in } V\right\} .
$$

This set $M(V)$ can be split into equivalence classes $r_{1}, . . r_{N}$ of loops that are homotopic with respect to $\mathbb{R}^{2} \backslash\{x \in$ $\left.\mathbb{R}^{2} \mid V(x)=0\right\}$. We define a vortex region candidate $R_{i}$ as the area that is covered by the lines of the equivalence class $r_{i}$.

The union of lines that wind around a common center define a vortex region candidate. A vortical behavior of the flow in such a region is not enforced by the definition. For a restriction to vortex regions, an additional filtering step must be applied. Filter criteria can be based, e.g., on the presence of complex eigenvalues of the enclosed critical points, vorticity or a predominance of curl within a region.

We extract vortex region candidates bounded by loops that start and end at saddle points. Due to continuity, at least one saddle is included in the closure of a bounded region $R_{i}$. The vortex region candidates $R_{i}$ are disjunct but may be nested, thus introducing a spatial hierarchy.

\subsection{Extraction Algorithm}

We present an algorithm that extracts all bounded vortex region candidates of a 2D vector field by detecting the boundary loops of these regions, i.e. loops that start and end at the saddle points of the vector field.

The extraction algorithm is based on intersection testing: Given a positively oriented boundary loop $\gamma_{\phi}$ with a single critical point inside, as illustrated in Fig. 4 . In $V_{\phi}, \gamma_{\phi}$ is a streamline. While further rotating the vector field up to $\pi$, the vectors of $V_{\psi}$ with $\psi \in(\phi, \phi+\pi)$ on $\gamma_{\phi}$ point inside, i.e., streamlines of $V_{\psi}$ can enter $\gamma_{\phi}$, but not leave it. Thus, two 


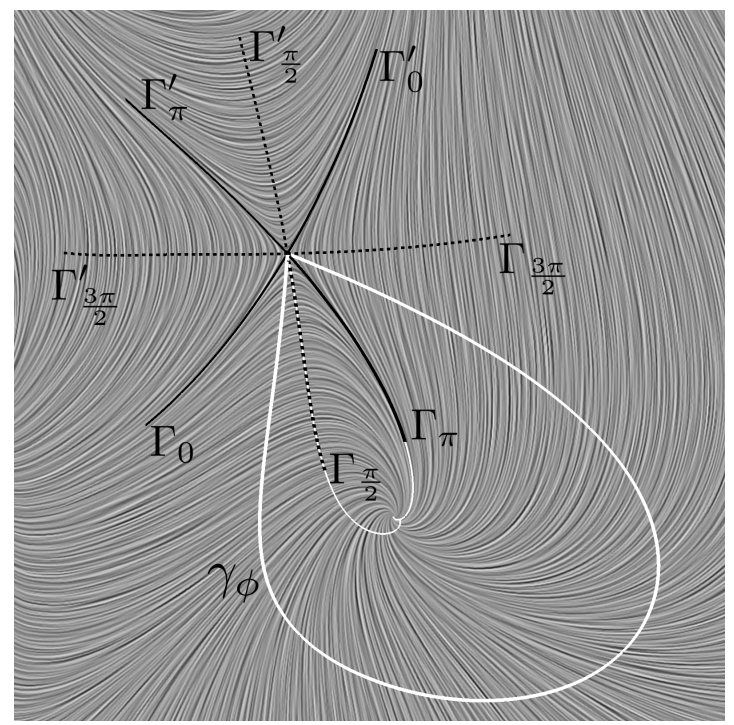

Figure 4: The LIC visualization in the background depicts the streamlines of some vector field $V$. The white line $\gamma_{\phi}$ is a closed streamline in the rotated vector field $V_{\phi}$. Eight streamlines $\Gamma_{\frac{k \pi}{2}}$ and $\Gamma_{\frac{k \pi}{2}}^{\prime}$ are simultaneously tracked in $V_{\frac{k \pi}{2}}$, $(k=0, \ldots, 3)$. In this example the streamlines $\Gamma_{\frac{\pi}{2}}$ and $\Gamma_{\pi}$ cannot cross the white line, so they necessarily intersect, or both end at the same critical point in the center of the region.

streamlines started from the saddle of two differently rotated vector fields in $(\phi, \phi+\pi)$ must intersect inside. Sampling vector field rotations in steps of $\frac{\pi}{2}$ and tracing streamlines from the saddle as depicted, the streamlines $\Gamma_{\frac{\pi}{2}}, \Gamma_{\pi}$ traced in $V_{\frac{\pi}{2}}, V_{\pi}$ intersect. The angle difference can be extended up to $\pi$, while the streamlines still intersect. This way, the loop $\gamma_{\phi}$ is detected.

The extraction algorithm is outlined in Alg. 1. The search for boundary loops is performed from all saddle points of the vector field repeatedly. During the search, boundary loops are detected and previously detected boundary loops are clustered. The clustering is necessary for the detection of loops that encompass other vortex region candidates. The algorithm terminates when no additional boundary loops are found and the clustering did not change in a full pass over all saddles.

Initially, the set directions is filled with all search directions from all the saddle points of the vector field as illustrated in Fig. 4. Clustering is maintained by the id data structure. Initially, each saddle belongs to a different cluster. Later on, the merging of clusters is done (line 17) by declaring two clusters as equivalent.

For an element of directions, a search is performed in searchLoop. This method either detects a new boundary loop $\Gamma$ (line 12), the need for an update of the clustering

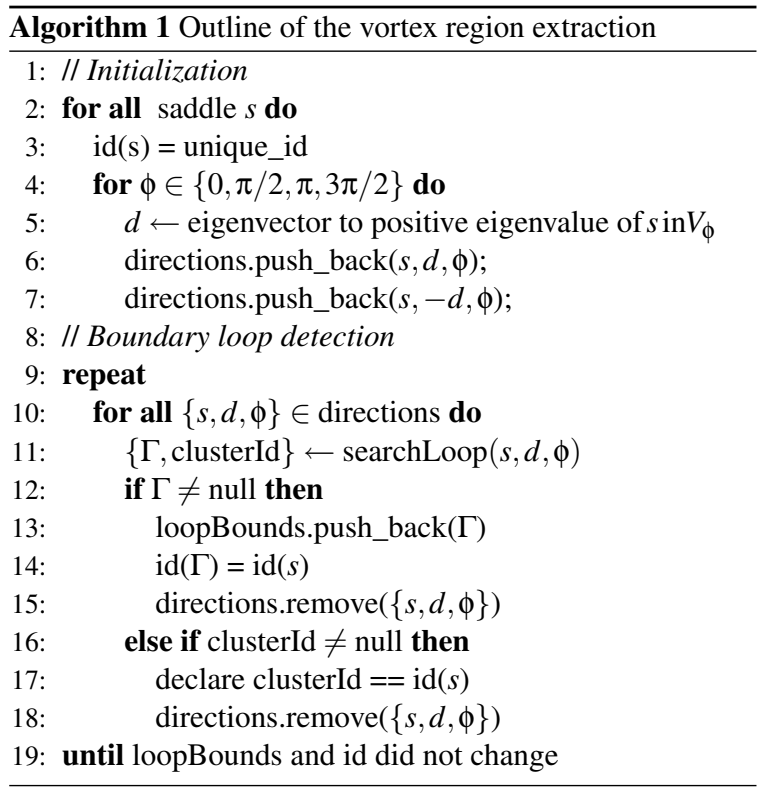

(line 16), or none of them. If a loop is found, it is added to the result set loopBounds and assigned to the same cluster as the saddle it starts from. If the clustering needs an update, the cluster of the saddle is merged with the other cluster. In both cases, the direction needs no further processing.

In searchLoop, the search starts from a saddle $s$, with direction $d$ and the field $V_{\phi}$. Initially with $\psi_{-}=\phi$ and $\psi_{+}=\phi+\frac{\pi}{2}$, two streamlines are traced in $V_{\psi_{-}}, V_{\psi_{+}}$and it is checked whether

1. the two streamlines intersect,

2. both streamlines intersect previously extracted boundary loops that belong to the same cluster.

While one of the conditions is met for $\psi_{-}$and $\psi_{+}$, the angle difference is extended in steps of $\frac{\pi}{2^{i}}, i=2, \ldots N$ up to an angle close to $\pi$ by testing streamlines for the angles $\left\{\psi_{-}-\frac{\pi}{2^{i}}, \psi_{+}\right\}$and $\left\{\psi_{-}, \psi_{+}+\frac{\pi}{2^{i}}\right\}$. If one of them satisfies the conditions, new angles $\psi_{-}, \psi_{+}$are obtained, otherwise the search failed. In practice, $N=8$ proved to be sufficient for all our examples.

If condition 1 was met in the last iteration, a new boundary loop $\Gamma$ is composed of the two last streamlines. If condition 2 was met, the cluster that was intersected by the two last streamlines needs to be merged with the cluster of the saddle.

Streamline integration always starts at a saddle point in direction of the eigenvector to the positive eigenvalue of the vector field. As this direction is ambiguous with respect to orientation, the direction must be specified. Two streamlines in $V_{\psi_{-}}, V_{\psi_{+}}$with $\psi_{-} \leq \phi \leq \psi_{+}$are always traced simultaneously. If the specified direction for $V_{\phi}$ is $d$, the eigenvector orientation for $V_{\psi_{+}}$is chosen such that continuously increasing the angle from $\phi$ to $\psi_{+}$would also continuously trans- 


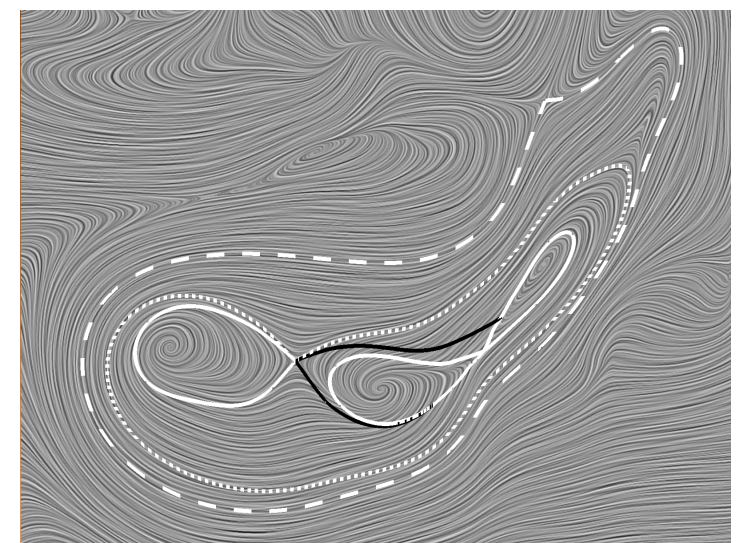

Figure 5: Closed lines that are detected from 3 saddle points. The 3 solid white boundary loops are detected directly. The black line consists of two streamlines that end at the same cluster, and thus clusters the 3 solid loops. The outer dashed line is detected after that clustering, and finally the inner dotted line.

form that direction. This is achieved by choosing the eigenvector $e_{+}$of $V_{\psi_{+}}$such that $d \times e_{+}>0$. The orientation of the eigenvector for $V_{\psi_{-}}$is chosen analogously, e.g. $e_{-} \times d>0$.

In Fig. 5 an example of the algorithm is given. The boundary loops of the vortex region candidates are the result of the extraction algorithm. The clustering is an internal support structure, needed for the detection of boundary loops that enclose other vortex region candidates.

\section{Results}

In Fig. 3(a) a complex shaped vortex region extracted with the proposed algorithm is depicted. The shape of the region is neither convex nor star-shaped, but has a smooth outer boundary. The field with non-vanishing divergence exhibits streamlines converging towards the critical point in the center. Applied to slices in a 3D velocity field, in Fig. 6, contour lines of the tip vortices of a rotating ship propeller are extracted. Note that the velocity field projected onto a slice is not divergence-free.

A hierarchy of vortex regions is displayed in Fig. 1. In Fig. 7 a vortex splitting event is shown in six snapshots of a time-dependent simulation of the Kármán vortex street. It depicts the flow situation directly behind a cylinder. The large vortex on the lower part of the first image splits into two vortices. One of them remains, the other separates and moves downwards the flow. During the splitting, two inner vortices emerge. They grow within the outer vortex region until they fill the outer space and the two vortices are completely split.

For comparison of our results to methods based on starshaped regions, we refer to Fig. 8 . The methods compute the

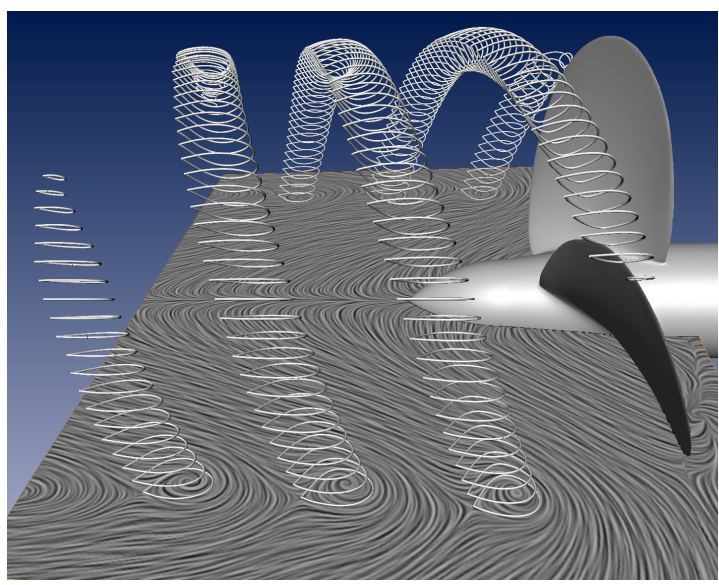

Figure 6: Tip vortices of a ship propeller are visualized as boundary contours of $2 D$ vortex regions of the velocity field projected onto planes rotated around the axis of the ship propeller. The LIC image depicts such a projected $2 D$ velocity field. Note that such a field is not divergence free.

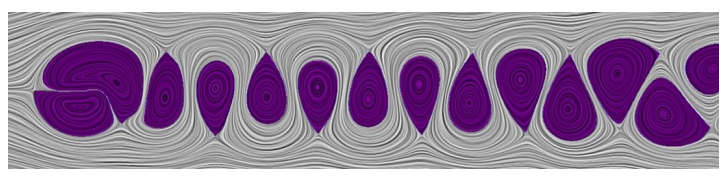

(a) Our method

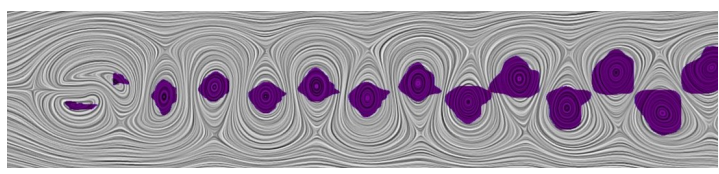

(b) $\lambda_{2}<0$ [SRE05]

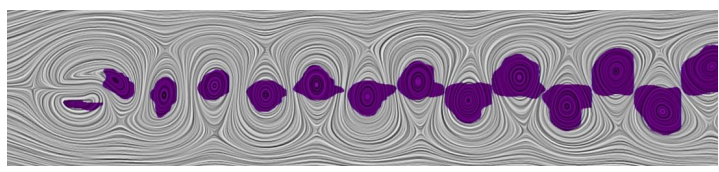

(c) Rankine vortex model [GTS*04]

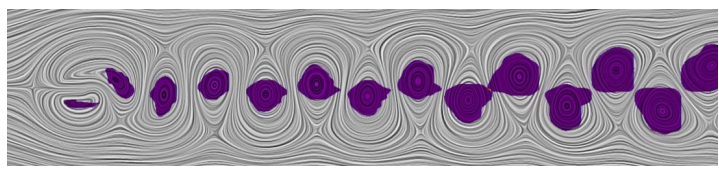

(d) Vortex strength [BPSS02]

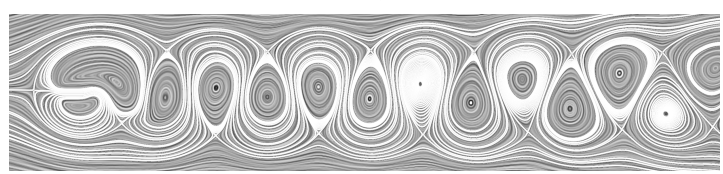

(e) Vector field topology

Figure 8: Comparison of our method (a) to other methods $(b)-(d)$ and to vector field topology $(e)$. Star-shaped regions are extracted and smoothed according to the indicated method. The displayed slice of the data set consists of $140 \times 45$ vectors. 

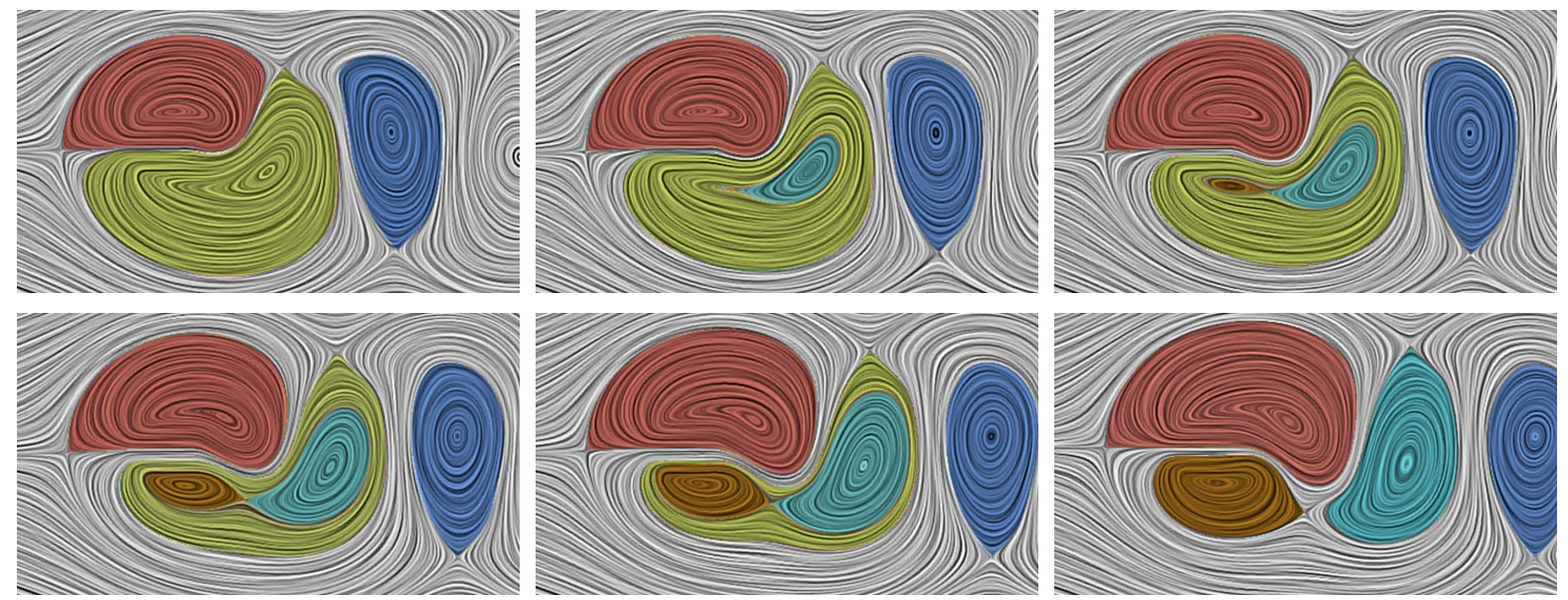

Figure 7: Extracted vortex regions in snapshots of a time-dependent flow behind a cylinder showing a vortex splitting event. Very thin regions around inner vortices are due to small divergence of the field.

vortex extent in planes for given vortex cores. Star-shaped regions are extracted based on thresholds of $\lambda_{2}$ [SRE05], the Rankine vortex model [GTS* 04 ] and regions with complex eigenvalues of the velocity gradient tensor [BPSS02]. The boundaries of these regions are smoothed, but still are irregularly shaped. The regions extracted with our method are larger compared to the other techniques.

A comparison to the geometric approach of Sadarjoen and Post [SP00] is presented in Fig. 10, using the same data set. Our approach handles nested vortex structures explicitly. In the algorithm of Sadarjoen and Post it depends on parameters of the clustering algorithm whether two nearby vortices overlap, as it is the case e.g. on the right side, where ellipses interfere. In this vector field with elongated and weak vortices, the hierarchy of vortex regions is especially useful. Both structures, small-scale inner vortex regions and largescale outer vortex regions are extracted and displayed simultaneously.

In Fig. 9 the extracted vortex regions are related to a physical definition of vortex regions in the style of the Rankine vortex model. In this model the maximum tangential velocity defines the extent of a vortex. We computed the average tangential velocity for all loops of a vortex region and used the loop with maximum average velocity to define a Rankine vortex region.

Using non-optimized software, the extraction of 500 vortex regions in a field of $64 \times 64$ randomly generated vectors takes 10 seconds on a laptop computer.

\section{Discussion}

Due to the ability of the presented method to extract vortex regions hierarchically, the dependency of nested vortices is explicitly known. This allows a viewer to see vortex merging or splitting events even in a single time step of a timedependent vector field. For example, a breaking vortex can dominate, but also have smaller vortices inside, pointing to transitional states, as exemplified in Fig. 7. This hierarchy can be exploited for visually tracking such events in timedependent flows. The detection of a hierarchy is an integral part of our method, which is in contrast to the feature tracking architectures proposed by Samtaney et al. [SSZC94] and Reinders et al. [RPS01]. Therein the event-detection is separated from the feature extraction step.

The presented method is based on a purely geometrical definition. However, the results coincide better with an intuitive notion of a vortex region, as those based on physical quantities as $\lambda_{2}$, the Rankine vortex model or vortex strength as depicted in Fig. 8. One reason for this is, that our method aims to extract the maximum region of swirling flow, whereby physical founded definitions, e.g., a threshold of $\lambda_{2}<0$, often define the extent of a vortex core region, i.e., the center of swirling flow.

Because our definition for vortex regions is based on vector field topology, and therefore not Galilean invariant, the frame of reference must be carefully chosen. This can be mitigated by pre-processing the flow field to reveal the features of interest. For example, if the velocity of the vortex motion is known in advance, it can be subtracted. A general method for unknown vortex motion is presented by Wiebel et al. [WGS05], who propose to subtract the divergence- and curl-free potential flow field in a region of interest. Any solution that works for vector field topology works also for our approach.

The ability of our method to handle fields with divergence makes it directly applicable on 2D slices through a 3D field. The tangential component in such a slice is not divergence- 


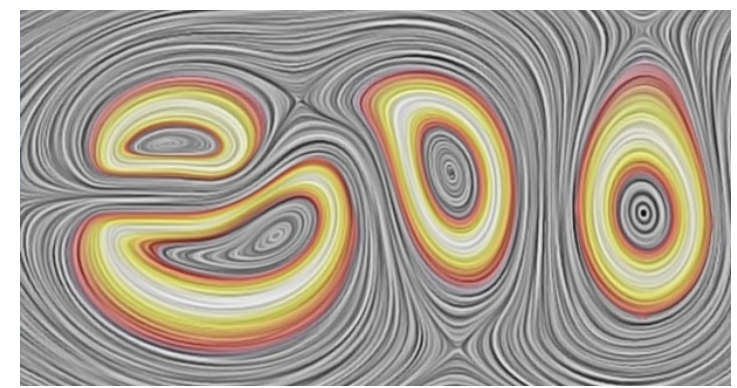

Figure 9: Outline of four Rankine based vortex regions. Color coded is the average tangential velocity along the loops of a vortex region. The white color denotes the local maximum, defining the Rankine based vortex region.

free due to fluid motion perpendicular to the slice, even for flows of incompressible fluids. Nonetheless, vortex regions can be identified. In practice, $2 \mathrm{D}$ slices through $3 \mathrm{D}$ flow fields are a useful tool, e.g. [GTWJ08, TGK*04].

The Rankine vortex model complements our vortex region definition in a reasonable way. It is build on loops, e.g. closed streamlines in rotated vector fields, and thus offering a natural way for the definition of a tangential direction. Those loops can be found at every point of a vortex region. By choosing the loop with the maximum average tangential velocity, the Rankine vortex regions are extracted. The approach of Garth et al. [GTS*04] is based on the assumption of circular vortices, as the direction perpendicular to the emitted ray is used as tangential direction. The extracted regions are non-smooth. Smoothing is done in postprocessing. In contrast, our vortex regions are constructed by integrating the vector field, yielding regions that are an order smoother as the interpolation of the original data values.

We assume to have only first-order critical points, but it should be possible to extend our method such that it can handle higher order singularities. Our extraction starts from the saddle points of a vector field and uses the directions of the separatrices to guide the search. Vector fields with higher order singularities have similar topological structures, as discussed by Scheuermann et al. [SKMR98]. Given the separatrices of such vector fields, the main idea of the extraction algorithm, the sampling of directions with angles of $\frac{\pi}{2}$, could be directly applied to those vector fields.

The proposed algorithm detects all bounded regions that end at the saddles of a vector field. In the current version regions that touch the domain boundary are not detected automatically. Including the boundary switching points of the vector field into the algorithm would improve the method to detect regions that touch the boundaries. Note, though, that boundary switching points move along the boundary if the vector field is rotated, which might have some unforeseen implications.

\section{Conclusion and Future Work}

We have introduced a method for extracting vortex regions that is consistently based on streamlines of swirling motion flow. The robust and parameter-free algorithm is able to extract complex-shaped vortex regions independently of the presence of divergence. Adjacent vortices are separated and hierarchical vortex structures in complex vortex systems can be revealed. The algorithm is grid-independent, up to routines for streamline tracing and critical point detection.

Our results suggest that the extracted vortex regions might be particularly useful for analyzing vortex dynamics on a structural level. Various applications are possible, e.g. measuring vortex sizes and strengths, monitoring splitting and merging events, or representing vortex evolution of timedependent flow fields in event graphs. Utilization of the method to real world applications needs to be investigated.

Another application is to utilize the knowledge gained with the presented method for enhancement of vector field topology depictions. Separatrices of vector fields tend to agglomerate in the vicinity of vortical regions. Explicit knowing of the vortex regions might help to avoid clutter there.

Although we have so far applied our region definition only to regions that exhibit some kind of swirling behavior, the restriction to swirling motion is not part of the definition of vortex region candidates. The extraction method could be applied in the same way for non-swirling regions, even for rotation-free gradient vector fields. The question then is, how to interpret the results.

\section{Acknowledgements}

This work was supported by the European Commission FP6 Project VIRTUE and by the German Research Foundation (SFB 557 "Control of complex turbulent shear flows"). We thank Frits Post and Ari Sadarjoen for the comparative figure of the Bay of Gdansk and the provision of the CFD data set. Further, W. Kollmann, University of California Davis for the provision of the swirling jet dataset. The INSEAN E779A propeller geometry has been provided by INSEAN, CFD simulation has been performed by HSVA.

\section{References}

[Asi93] Asimov D.: Notes on the Topology of Vector Fields and Flows. Tech. Rep. RNR-93-003, NASA Ames Research Center, 1993.

[BP02] Bauer D., Peikert R.: Vortex Tracking in Scale-Space. In Data Visualisation 2002 (Proceedings of the Joint Eurographics - IEEE TCVG Symposium on Visualization) (2002), Eurographics Association, pp. 233-240.

[BPSS02] Bauer D., Peikert R., Sato M., Sick M.: A case study in selective visualization of unsteady $3 \mathrm{~d}$ flow. In VIS ' 02 : Proceedings of the conference on Visualization '02 (Washington, DC, USA, 2002), IEEE Computer Society, pp. 525-528. 

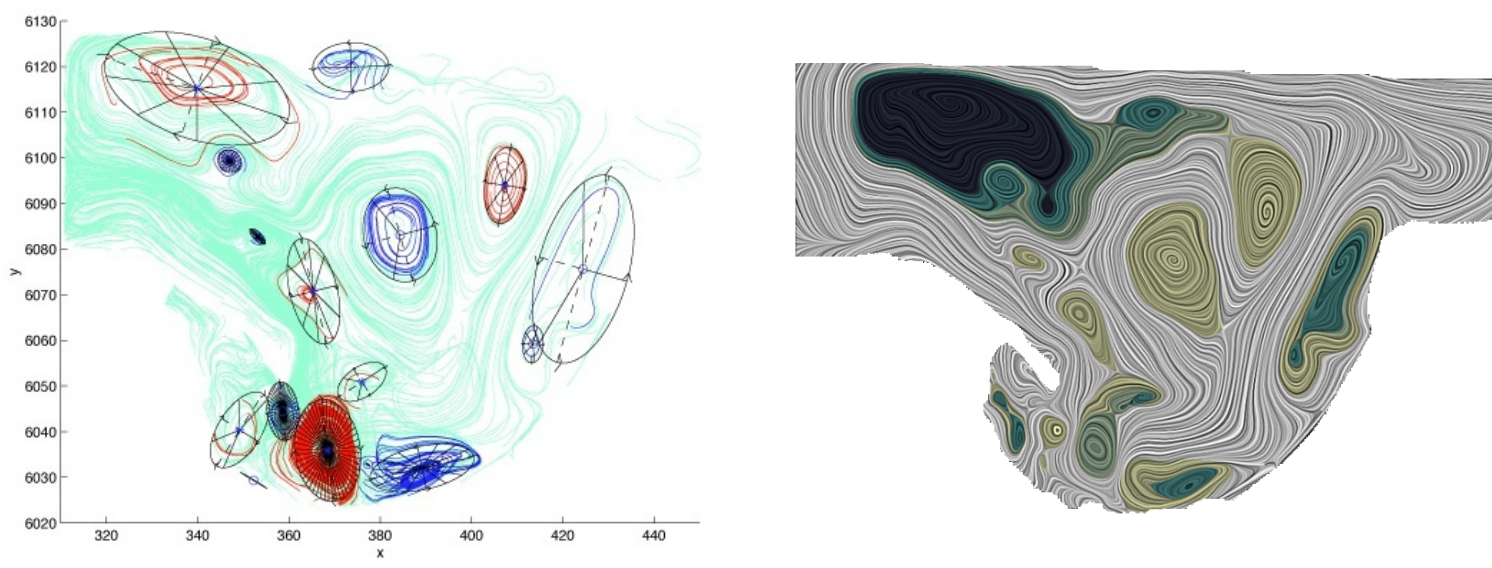

Figure 10: Comparison of the geometry based iconic vortex visualization of Sadarjoen and Post [SP00] to the hierarchical vortex regions. The depicted slices are slightly different. Sadarjoen and Post used a horizontal slice $(k=9)$ in the $(i, j, k)$ indexed parameter space; we use a horizontal slice in physical space.

[BS95] BANKS D. C., Singer B. A.: A predictor-corrector technique for visualizing unsteady flow. IEEE Transactions on Visualization and Computer Graphics 1, 2 (1995), 151-163.

[GTS*04] Garth C., Tricoche X., Salzbrunn T., Bobach T., SCHEUERMANN G.: Surface techniques for vortex visualization. In VisSym (2004), pp. 155-164, 346.

[GTWJ08] Garth C., Tricoche X., Wiebel A., Joy K. I.: On the role of domain-specific knowledge in the visualization of technical flows. In Proceedings of Simulation and Visualization 2008 (Feb. 2008), SCS Publishing House, pp. 107-120.

[Hel58] Helmholtz H.: Über Integrale der hydrodynamischen Gleichungen welche den Wirbelbewegungen entsprechen. $J$. Reine Angew. Math. 55 (1858), 25-55.

[HH89] Helman J., HeSselink L.: Representation and display of vector field topology in fluid flow data sets. Computer 22, 8 (Aug. 1989), 27-36.

[HH91] Helman J. L., Hesselink L.: Visualizing vector field topology in fluid flows. IEEE Comput. Graph. Appl. 11, 3 (1991), 36-46.

[Hun87] HunT J. C. R.: Vorticity and vortex dynamics in complex turbulent flows. In Canadian Society for Mechanical Engineering, Transactions (ISSN 0315-8977), vol. 11, no. 1, 1987, p. 21-35. (1987), vol. 11, pp. 21-35.

[JH95] JEONG J., HUSSAIN F.: On the identification of a vortex. Journal of Fluid Mechanics 285 (1995), 69-94.

[JKJTM06] Jankun-Kelly M., Jiang M., Thompson D., MACHIRAJU R.: Vortex visualization for practical engineering applications. IEEE Transactions on Visualization and Computer Graphics 12, 5 (2006), 957-964.

[PVH*03] Post F. H., Vrolijk B., Hauser H., Laramee R. S., Doleisch H.: The state of the art in flow visualisation: Feature extraction and tracking. Computer Graphics Forum 22, 4 (2003), 775-792.

[RPS01] REINDERS F., POST F., SPOELDER H.: Visualization of time-dependent data using feature tracking and event detection. The Visual Computer 17, 1 (February 2001), 55-71.

[RSVP02] Reinders F., SAdARJoen I., VRolijk B., Post F.: Vortex tracking and visualisation in a flow past a tapered cylinder. Computer Graphics Forum 21, 4 (11 2002), 675-682.
[SKMR98] Scheuermann G., KrÜger H., Menzel M., RocKWOOD A. P.: Visualizing nonlinear vector field topology. IEEE Transactions on Visualization and Computer Graphics 4, 2 (1998), 109-116.

[SP99] Sadarjoen I. A., Post F. H.: Geometric methods for vortex extraction. In Joint Eurographics-IEEE TVCG Symposium on Visualization (1999), pp. 53-62.

[SP00] SADARJoen I. A., Post F. H.: Detection, quantification, and tracking of vortices using streamline geometry. Computers \& Graphics 24, 3 (June 2000), 333-341.

[SRE05] Stegmaier S., Rist U., ERTL T.: Opening the Can of Worms: An Exploration Tool for Vortical Flows. In Proceedings of IEEE Visualization '05 (2005), C. Silva and E. Gröller and H. Rushmeier, (Ed.), IEEE, pp. 463-470.

[SSZC94] Samtaney R., Silver D., Zabusky N., CaO J.: Visualizing features and tracking their evolution. IEEE Computer 27, 7 (July 1994), 20-27.

[SWC*08] Schneider D., Wiebel A., Carr H., HlaWITSCHKA M., SCHEUERMANN G.: Interactive comparison of scalar fields based on largest contours with applications to flow visualization. IEEE Trans Vis Comput Graph 14, 6 (2008), 14751482 .

[TGK*04] Tricoche X., Garth C., Kindlmann G., Deines E., Scheuermann G., Ruetten M., Hansen C.: Visualization of intricate flow structures for vortex breakdown analysis. In Proceedings of IEEE Visualization 2004 (October 2004), pp. 187-192.

[TWhS04] Theisel H., Weinkauf T., Hege H.-C., Seidel H.-P.: Grid-independent detection of closed stream lines in 2D vector fields. In Proc. Vision, Modeling and Visualization 2004 (Stanford, U.S.A., November 2004), pp. 421-428.

[WGS05] Wiebel A., Garth C., Scheuermann G.: Localized flow analysis of 2d and 3d vector fields. In EuroVis (2005), Brodlie K., Duke D. J., Joy K. I., (Eds.), Eurographics Association, pp. 143-150.

[WS01] Wischgoll T., Scheuermann G.: Detection and visualization of closed streamlines in planar flows. IEEE Transactions on Visualization and Computer Graphics 7, 2 (2001), 165172. 